\title{
Case Report on Locked Pubic Symphysis with Concomitant Ipsilateral Acetabular Fracture
}

\section{Milkias Tsehaye $\mathbb{D}$ \\ Daniel Teklu}

Department of Orthopedic Surgery, St. Paul's Hospital Millennium Medical College, Addis Ababa, Ethiopia
Correspondence: Milkias Tsehaye Department of Orthopedic Surgery, St. Paul's Hospital Millennium Medical College, P.O. Box I27I, Addis Ababa, Ethiopia

Tel +25I9II370866

Email milkrody@gmail.com; milkias. tsehaye@sphmmc.edu.et
Background: Locked pubic symphysis or overlapping symphysis is a rare injury which happens when a pubic bone displaces behind the contra lateral pubic rami. It usually arises from lateral compression injuries. In this case report, we try to share our experience on the operative fixation of locked pubic symphysis and concomitant ipsilateral acetabular fracture. Case Presentation: A 24-year-old male patient presented after a road traffic accident with lateral compression injury to the pelvis. He was found to have locked pubic symphysis, right side transverse acetabular, and right side closed tibial fracture. He was taken to the operation theater 10 days later after he was managed for the additional chest contusion he had. Successful open reduction and plate fixation of the overlapped symphysis and percutaneous screw fixation of the ipsilateral acetabulum were done at the same time. An intramedullary nail was inserted for the tibial fracture.

Conclusion: Locked symphysis is a rare trauma and even more complex when associated with acetabular fracture. Both situations may happen at the same time and could be managed operatively.

Keywords: locked pubis symphysis, overlapping symphysis, acetabular fracture

\section{Introduction}

Among a variety of pelvic injuries stated by the Young and Burgess classification, lateral compression is the most common. ${ }^{12}$ Of the lateral pelvic injury patterns the locked pubis symphysis is a very rare but serious injury. This unique injury is characterized by overlapping pubic symphysis disruption, the pubic bone on one side entrapped behind or very rarely anterior to the contra lateral pubic rami. ${ }^{1-9}$ Only a very small proportion of pelvic trauma resulting from lateral compression injuries end up as locked pubic symphysis injuries. ${ }^{1}$

A locked pubic symphysis is quiet rare injury and even more when associated with acetabular fracture. It was first described by Eggers in 1952 (in Britain). ${ }^{11}$ To the best of our knowledge this is the first case report in Ethiopia.

\section{Case Presentation}

Our patient is a 24 year old male who was involved in a motor vehicle accident. He was an unrestrained occupant in the front seat of a truck when he sustained the injury. Upon arrival to our hospital, he was tachycardic and tachypneic and was managed accordingly with ATLS protocol.

He had severe pain over the pelvis and gross deformity over the the right leg with distal neurovascular structures intact on examination. He had degloving soft 
tissue injury over the right heel for which debridement was done at the hospital where he was referred from.

Pelvis X-ray and CT scan was taken and showed right side overlapping symphysis pubis associated with ipsilateral transverse acetabular fracture type (Figure 1). The $\mathrm{x}$-ray of the right leg showed a closed tibial shaft fracture.

The patient was unable to maintain his $\mathrm{O}_{2}$ saturation with atmospheric breathing at the emergency room. He was put on intranasal $\mathrm{O}_{2}$ support for a lung contusion injury he had. He had no urinary complaint or blood at the urethral meatus, therefore a Foley catheter was inserted with no need for cystourethrography. He was taken to the operating theater 10 days after the injury. Closed reduction of the locked symphysis was not attempted inorder to avoid further displacment of the ipsilateral acetabular fracture. The patient was positioned supine on the operating table and his pelvis and both legs were prepped and draped free. The symphysis pubis was approached through a Pfannenstiel incision $2 \mathrm{~cm}$ above the pubic tubercle with about a $10 \mathrm{~cm}$ incision width. The rectus abdominis was found to be partially contused and was further surgically splitted at the midline vertically to retract it laterally. The retropubic space was accessed with blunt dissection to expose the overlapped pubis symphysis and the rami (Figure 2).

Then 3.5 cortical screws were applied on the opposing pubic bone bodies with an anterior to posterior direction, approximately $2 \mathrm{~cm}$ lateral from the center using them as a joystick to apply a reduction clamp. Using the Jung Bluth clamp on the inserted screws, rotational and disimpacting force was applied and reduction of the symphysis pubis was achieved. After achieving reduction, $3.5 \mathrm{~mm}$ size and 6-hole precontoured reconstruction plate was applied on the superior aspect of the symphysis. The plate was secured with three cortical screws on each side.

For the percutaneous fixation of the acetabulum, an entry point was identified laterally on the supra-acetabular area with the help of a fluoroscopy image aiming to the symphysis and a stab incision was made for a guide wire insertion. The guide wire was directed from the entry point towards the symphysis using the pelvic inlet and obturator oblique outlet views under fluoroscopy. ${ }^{10}$ A 4.5 cannulated drill bit was used to create a path for the screw. Then the transverse column acetabular fracture was fixed percutaneously, using a $6.5 \mathrm{~mm}$ size and $100 \mathrm{~mm}$ length partially threaded cannulated screw. A post-operative x-ray image was taken the day after surgery (Figure 3).

Post-operatively, the patient was stable and followed in the ward with 72 hours of post-operative broad spectrum intravenous antibiotics and adequate analgesics. Prophylactic unfractionated heparin 7,500 IU subcutaneous twice a day was continued according to the hospital's protocol.

The patient was discharged after 5 days of hospital stay, shifting the UFH to oral rivaroxaban $10 \mathrm{mg}$ per day for 1 month and additional adequate analgesics. He had no access for center-based physiotherapy, hence was advised on home physiotherapy with hip and knee passive range of motion and non-weight bearing status of 6 weeks period due to the associated tibial fracture he had. On the 2 nd week of his visit to the OPD, the surgical wound was in good condition and the stitches were removed. He was on bilateral axillary

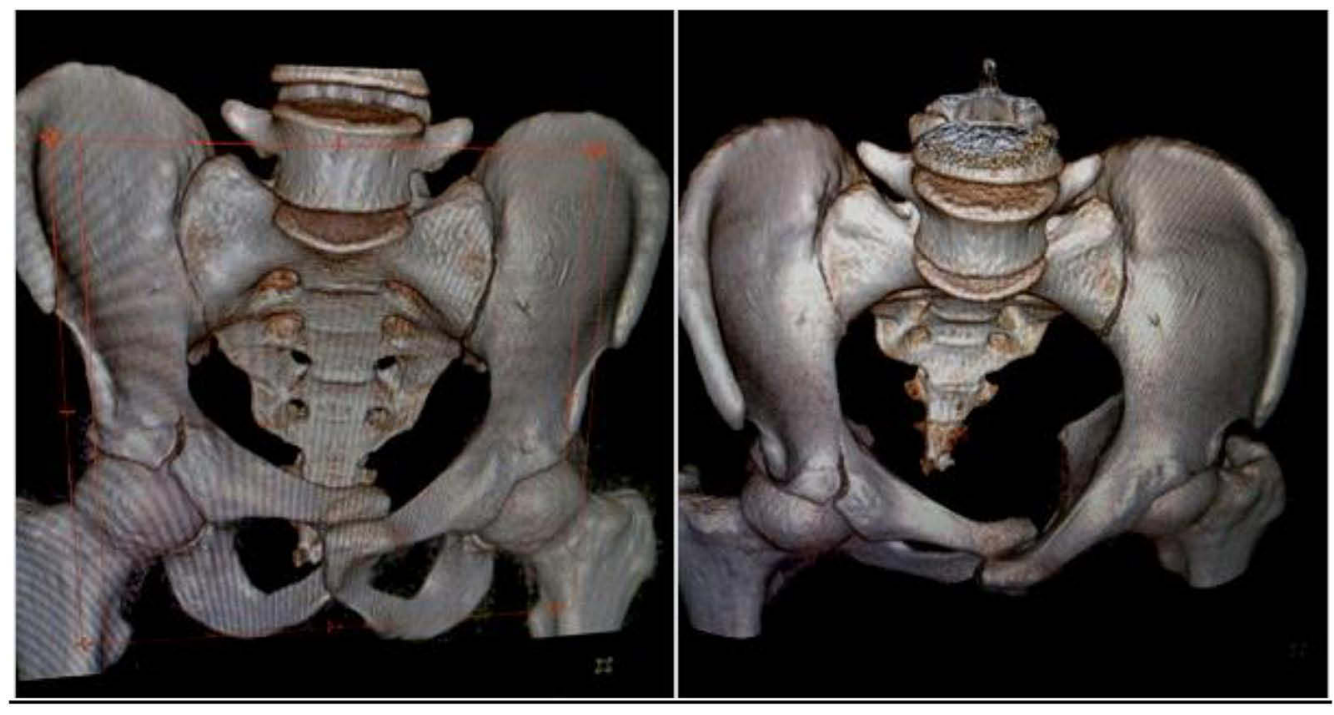

Figure I 3D CT showing the right pubic bone locked behind the left pubic rami. The pelvis also has a right transverse acetabular fracture. 


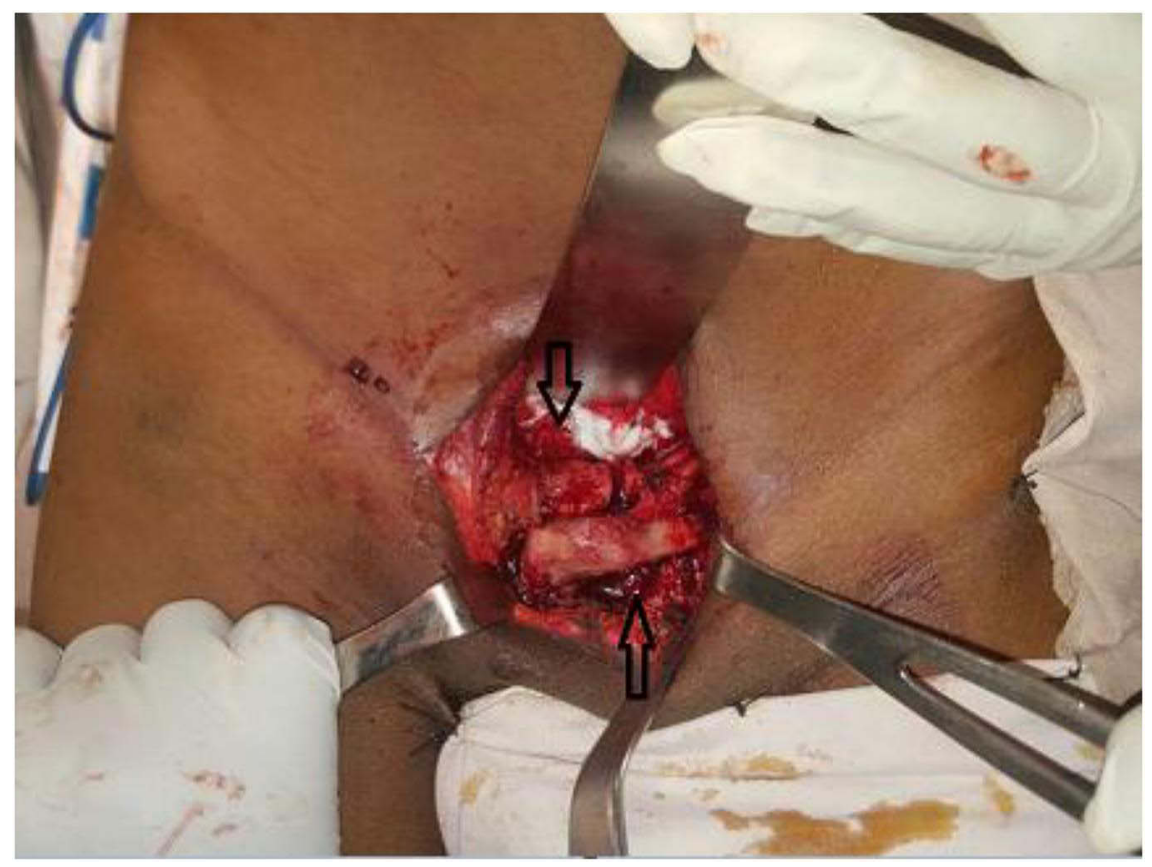

Figure 2 Shows x-ray of the overlapped symphysis, the upper arrow shows the displaced right side pubic bone behind the left pubic bone (lower arrow).
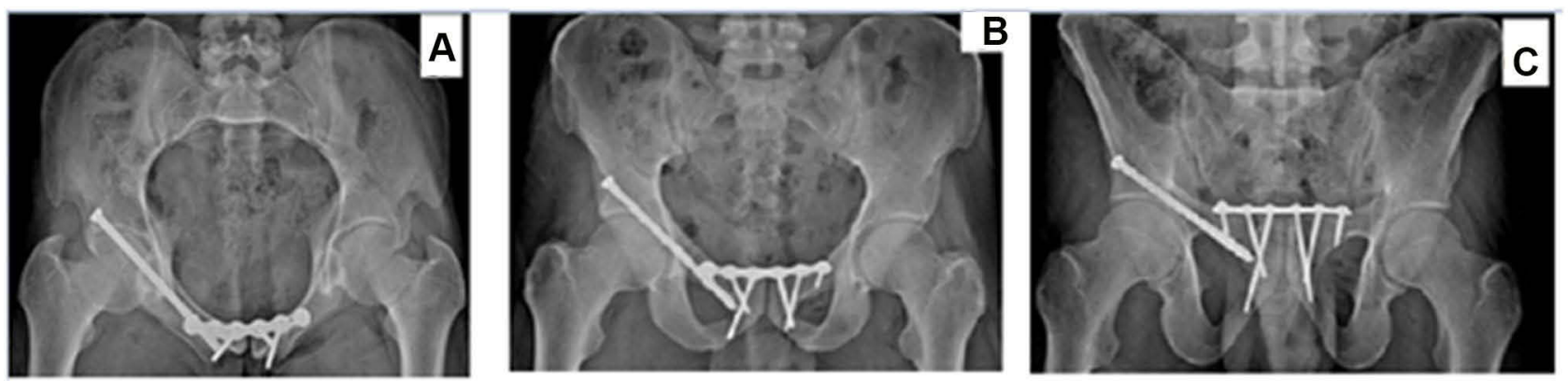

Figure 3 The post-op x-ray after fixation of the symphysis pubis with plating and right acetabulum with lag screw. (A) Inlet pelvic (B), Anteroposterior pelvic (C), Outlet pelvic $\mathrm{x}$-rays.

crutch support for bed to chair and home mobilization during the first 6 weeks of his post-operation period. At his 2-month follow-up, the patient had no pelvic pain and was able to partially weight bear with the help of a crutch. He had no urinary symptoms or suprapubic pain. At 6-months follow-up he was back to his normal pre-injury activity. Pelvic radiograph showed the hardware in a good position with fracture and dislocations staying reduced (Figure 4).

\section{Discussion}

Locked or overlapping symphysis pubis is lateral compression injury where the intact pubis on one side moves behind or rarely in front of the contralateral intact pubis. ${ }^{1-9}$ It is caused by hyperextension and adduction forces on the hip and femur of the affected side. Theoretically, internal or external rotation of the femur displaces the pubic bone posterior or anterior to the contra lateral intact pubic body. ${ }^{1}$ It is a rare injury, not more than 30 case reports written in English language literature on this particular case. To our knowledge, we have not found any case report written about simultaneous presentation of a locked symphysis case and acetabular fracture combined.

Males are more affected than women $(80 \%$ found in males according to Afshar et al $^{1}$ after reviewing 25 patients in 20 case reports). The common age group for this injury is usually between $16-45$ years of age, and the commonest mechanism is motor vehicle accident.

Urethral injury is commonly associated in patients with locked symphysis pubis, usually manifesting with 


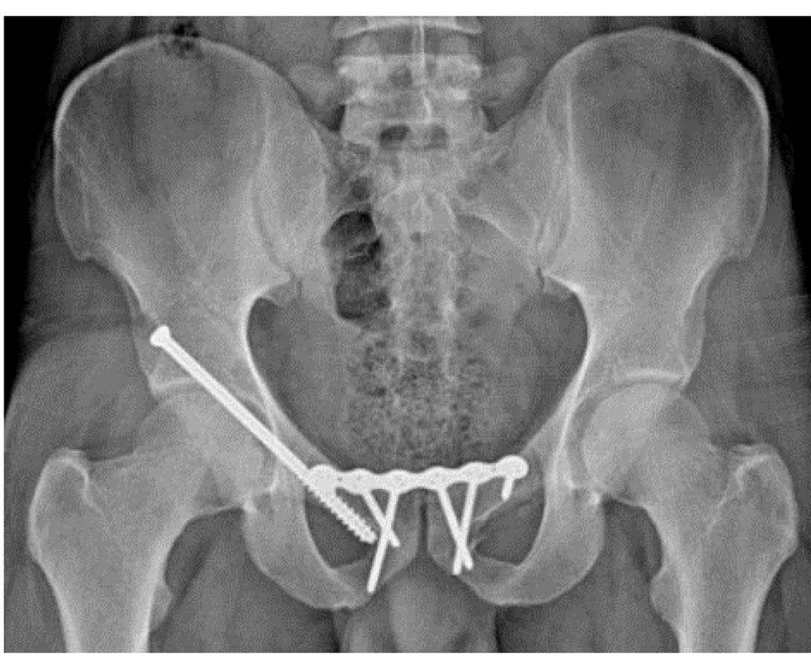

Figure 4 Anteroposterior x-ray image of the pelvis at 6 months follow up.

a distended bladder and blood at the urethral meatus. ${ }^{1,5,6}$ Another commonly associated injury is an injury to the posterior pelvic ring structure which could be either ligamentous or fracture on the sacral bone. ${ }^{2,5,6}$

The locked pubis symphysis might better fit under the 61-B2 with AO classification.

Chee et $\mathrm{al}^{2}$ were the latest to propose a classification based on the severity of the overlap and involvement of the posterior ring, suggesting management for each grade. Grade I is an overlapping pubic bone within the width of the pubic body, Grade II is overlapping of the pubic bone beyond the width of the pubic body, Grade III is an incarceration of pubic symphysis on the opposite side obturator foramen. It is further classified as $\mathrm{A} / \mathrm{B}$ whether the posterior ring is involved or not. Grade IA and IIA injuries warrant an attempt of closed reduction, while Grade IB, IIB, and III warrant an open reduction and fixation.

The commonest classification scheme for acetabulum fractures is the Judet and Letournel system. There are 10 fractures or fracture combinations which are classified into five elementary and five associated fractures. The five elementary fracture types are posterior wall, anterior wall, posterior column, anterior column, and transverse. The five associated fracture types are combinations or partial combinations of the elementary fractures and include transverse with posterior wall, posterior column with posterior wall, T-shaped, anterior column or wall with posterior hemi transverse, and associated both column.

Closed reduction is the first line of management for locked pubis symphysis. It is usually done by reversing the proposed mechanism of the injury by flexing, abducting, and externally rotating the hip. This maneuver tightens the iliofemoral ligament and gentle rocking movement using the femur then may unlock the symphysis pubis. A stabilizing force on the contralateral iliac crest should be applied while trying the reduction. ${ }^{1,5,7}$

Open reduction would be the choice for overlapped/ locked symphysis pubis when the closed technique fails. It involves open visualization of the overlap through an anterior approach and reduction is attempted directly using different techniques. In most of the cases reported, reduction is achieved by conventional methods. ${ }^{5,7,8} \mathrm{In}$ some difficult cases, it might need an osteotomy of the undisplaced superior pubic ramus to achieve reduction. ${ }^{4}$ Stabilization is the next step after reduction and plating is the frequently preferred method.

\section{Conclusion}

Locked symphysis pubis is rare injury. It can occur associated with different soft tissue injuries. Though sacral fracture is the usual associated bony injury, it may occur also associated with an acetabular fracture. Open reduction and stabilization of the locked symphysis followed by fixation of the acetabular injury has good outcome.

\section{Ethics and Consent}

The patient agreed to give written informed consent to publish his case detail and accompanying images as well. Since it is a case report, approval from the institutional review board is not required.

\section{Disclosure}

The authors declare no conflicts of interest in this work.

\section{References}

1. Afshar A, Koushkzari M. Overlapped pubic symphysis; a case report and review of the literature. Arch Bone Jt Surg. 2015;3(3):212.

2. Chee WH, Ahmad AR. Locked pubic symphysis: a case report and review of literature. J Health Transl Med. 2018;21(2).

3. Cannada LK, Reinert CM. Case report: locked pubic symphysis: an open reduction technique. Clin Orthop Relat Res. 2009;467 (8):21922195.

4. Li K, Sun B, Zhu Y, Long H. Locked pubic symphysis into the obturator foramen: a rare case presentation and literature review. Orthop Traumatol Surg Res. 2013;99(1):106-110.

5. Thulasiraman V, Pandian TRR, Ashok S. Locked pubic symphysis-a case series. Inj Extra. 2010;41(2):20-24. doi:10.1016/j.injury.2009. 11.009

6. Fola $\mathrm{O}$, Guifo M, Tsiagadigui J, et al. Locked book pubic symphysis: a case report in a resource-limited setting in subSahara Africa. Int J Surg Case Rep. 2019;64:24-27. doi:10.1016/j.ijscr.2019.09.019 
7. Wuei Huang W. A case of locked pelvis, with no urogenital 0r vascular injury. Biomed J Sci Tech Res. 2018;3(5):3629-3631. doi:10.26717/BJSTR.2018.03.000977

8. Sreesobh K, Sageer A, Raffic M. Locked overlapping dislocation of the pubic symphysis into the obturator foramen: a case report. J Orthop Surg. 2006;14(2):200-203. doi:10.1177/230949900601400219

9. Maqungo S, Koller I, Roche S. Overlapping pubic symphysis dislocation: a case report and proposal of a classification system. $S A$ Orthop J. 2010;9(4):74-77.

10. Tile M, Helfet DL, Kellam JF, Vrahas M. Fractures of the Pelvis and Acetabulum, Principles and Methods of Management-Fourth Edition. Thieme Medical Publishers. 2015:626-627. doi:10.1055/b003-121618
11. Eggers GWN. Dislocations of the os coxae. Am J Surg. 1952;83 (3):300-307. doi:10.1016/0002-9610(52)90261-4

12. Burgess AR, Eastridge BJ, Young JW, Ellison TS, Ellison PS, Poka A. Pelvic ring disruptions: effective classification system and treatment protocols. J Trauma. 1990;30(7):848-856. doi:10.1097/ 00005373-199007000-00015

\section{Publish your work in this journal}

The International Medical Case Reports Journal is an international, peer-reviewed open-access journal publishing original case reports from all medical specialties. Previously unpublished medical posters are also accepted relating to any area of clinical or preclinica science. Submissions should not normally exceed 2,000 words or 4 published pages including figures, diagrams and references. The manuscript management system is completely online and includes a very quick and fair peer-review system, which is all easy to use. Visit http://www.dovepress.com/testimonials.php to read real quotes from published authors. 